\title{
The Use of Topical Local Anaesthetic Spray for Suturing Paediatric Intra-oral Lacerations
}

\author{
Karl F. B. Payne ${ }^{1} \cdot$ Punam Kalirai $^{2} \cdot$ Vijay Thirunavukarasu $^{2}$
}

Received: 15 June 2015 / Accepted: 18 March 2016/Published online: 17 May 2016

(C) The Association of Oral and Maxillofacial Surgeons of India 2016

\section{Dear Sir,}

We would like raise a point for discussion with regards to the use of topical local anaesthetic (LA) agents when suturing intra-oral lacerations in paediatric patients. It is often a difficult decision of how best to achieve anaesthesia in a paediatric patient with a simple intra-oral laceration. Due to poor patient compliance, unfortunately, it is commonplace to use either sedation or a general anaesthetic.

We would like to advocate the use of a topical LA spray when treating such lacerations. To-date we have used this method successfully on both tongue and lip paediatric intra-oral lacerations. In this method, 1-2 applications of Xylocaine Spray ${ }^{\circledR}$ (Fig. 1) are allowed to sit on the mucosa for up to $5 \mathrm{~min}$, achieving effective anaesthesia to allow for simple interrupted suturing. In our experience this appears to be more tolerable than LA infiltration, reducing patient distress and the need for possible increased intervention to achieve anaesthesia. We can find no recommendations precluding its use in this manner.

The efficacy of LA solutions to treat paediatric face and scalp lacerations has been previously demonstrated [1], but we are unable to find any literature that discusses the use of LA spray intra-orally. We accept that this method is limited to small simple lacerations only, however we would be interested to hear if other clinicians have adopted a similar technique.

Karl F. B. Payne

karlpayne@nhs.net

1 Oral and Maxillofacial Surgery Theme, Russells Hall

Hospital, Dudley, UK

2 ENT Surgery, Russells Hall Hospital, Dudley, UK

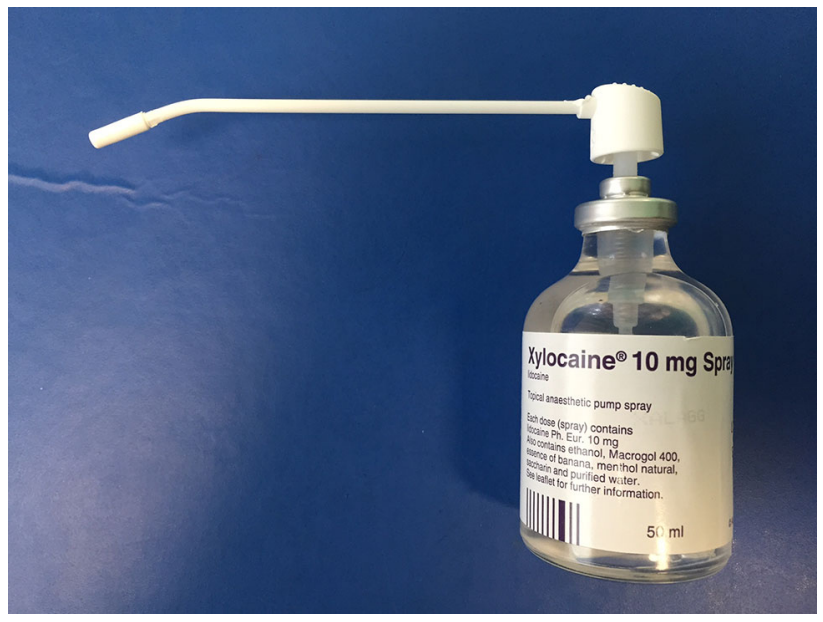

Fig. 1 Xylocaine Spray ${ }^{\circledR}$ (Astra Zeneca Ltd UK) with nozzle attached. Each spray delivers $10 \mathrm{mg}$ of lidocaine

Compliance with ethical standards

Conflict of interest None.

\section{Reference}

1. Resch K, Schilling C, Borchert BD, Kltazko M, Uden D (1998) Topical anesthesia for paediatric lacerations: a randomised trial of lidocaine-epinephrine-tetracaine solution versus gel. Ann Emerg Med 32(6):693-697 